\title{
Space-Based Photometry of Eclipsing Binaries
}

\author{
John Southworth \\ Astrophysics Group, Keele University, Staffordshire, ST5 5BG, UK \\ email: jkt@astro.keele.ac.uk
}

\begin{abstract}
I briefly review the history and prospects for the study of eclipsing binary star systems from space-based observatories. The benefits of shifting to space satellites lie in the high precision and cadence achievable, as well as the ability to access wavelength regions which are unattainable from the ground. Whilst small amounts of data on eclipsing binaries were obtained by the Voyager, IUE, OAO-II, Hipparcos and MOST, the more recent CoRoT and Kepler missions were the first to provide extensive data on large numbers of systems. The future holds the prospect of the PLATO satellite, which will go bigger, better and brighter.
\end{abstract}

Keywords. stars: binaries: eclipsing — stars: fundamental parameters

\section{Eclipsing binary stars from space}

The study of eclipsing binary stars rests on the analysis of extensive high-precision photometry. They are therefore natural candidates to be observed from space, where telescopes are free of the Earth's atmospheric scintillation, extinction, diurnal variations and weather. The resulting light curves can be of extremely high precision and cadence, and are able to spy into wavelength ranges which are inaccessible from the ground.

Early entrants into this field were the two Voyager missions, which both possessed ultraviolet imaging instruments among other capabilities, and the IUE and OAO-II satellites (e.g. Cherepashchuk et al., 1984; Kondo et al., 1994). The Hipparcos astrometry mission obtained photometry at several hundred epochs for many bright stars, discovering 343 new eclipsing binary systems (Perryman et al., 1997).

The WIRE satellite showed what was possible from space for bright stars. WIRE was launched into a polar orbit in March 1999 in order to obtain infrared photometry of galaxies, but an early failure of the coolant system rendered it unable to attempt its primary mission. Attention then shifted to its $5 \mathrm{~cm}$-aperture star tracker, which was in a position to obtain CCD photometry of bright stars at a cadence of $2 \mathrm{~Hz}$. Seven eclipsing binaries were observed which this instrument before communications were lost with the satellite (Bruntt \& Southworth, 2007, 2008). These included $\psi$ Centauri, a 4th-magnitude star which was not previously known to eclipse (Bruntt et al., 2006) and $\beta$ Aurigae, the first known and brightest eclipsing and spectroscopically double-lined binary system (Stebbins 1911, Southworth et al., 2007).

This work has subsequently been continued by MOST (Pribulla et al., 2010), CoRoT (e.g. Maceroni et al., 2009) and now Kepler. The Kepler satellite was launched in March 2009 and is dedicated to finding new extrasolar planets by the transit method (Borucki et al., 2010). The high-quality photometry it obtains is also excellent for performing asteroseismology and for studying eclipsing binaries. A small number of objects fit into both of these categories, and are of great prospect for furthering our understanding of the physics of stars. The Kepler light curve of KIC 10661783 (Fig. 1) shows it to be an oEA system: a semi-detached binary in which the primary star exhibits $\delta$ Scuti oscillations 


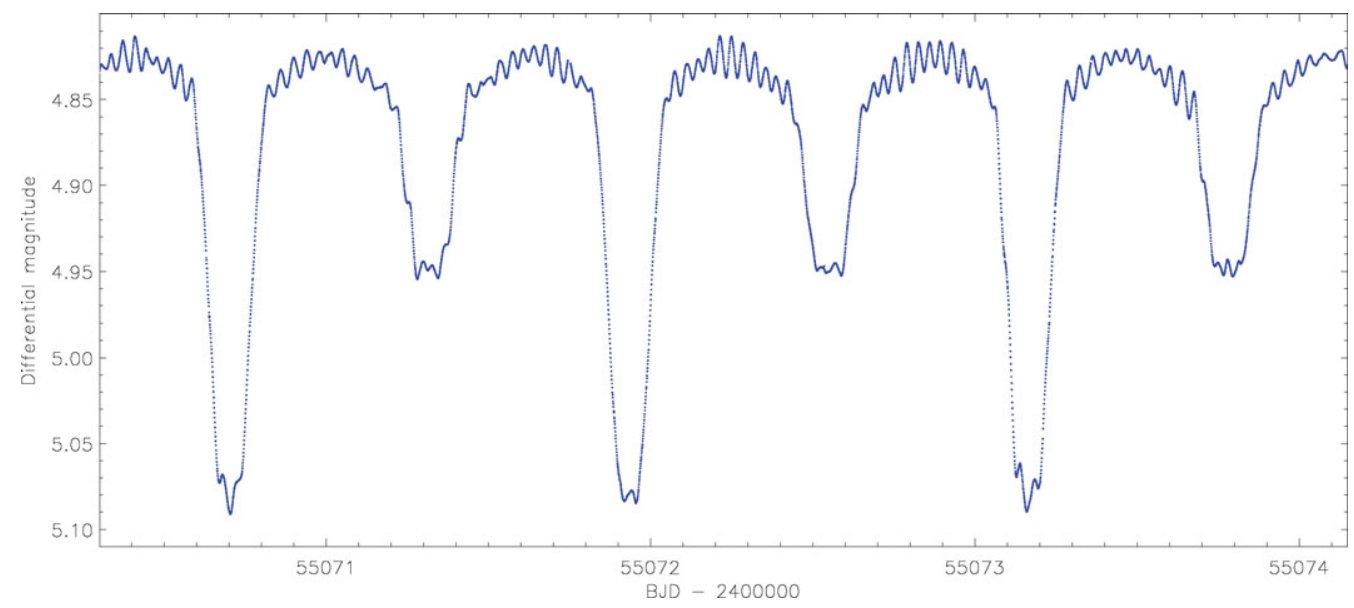

Figure 1. A small section of the Kepler satellite light curve of the oEA system KIC 10661783.

(Southworth et al., 2011). These data provided 58 oscillation frequencies, at which point we ran out of frequency resolution. Further observations have been obtained by Kepler and will certainly reveal a multitude of new pulsation modes in this system. Other examples include: KIC 8410638, an eclipsing binary containing a pulsating giant star and an F dwarf with an orbital period of at least 1 yr (Hekker et al., 2011); KIC 5952403, a hierarchical doubly-eclipsing triple system comprising a $\mathrm{G}$ giant in a $45 \mathrm{~d}$ orbit around a $0.9 \mathrm{~d}$ binary of two late-type dwarfs (Derekas et al., 2011); and the KIC 5897826 system of an F subgiant and two late-M dwarfs (Carter et al., 2011)

Whilst CoRoT and Kepler have furnished the cupboard with excellent light curves of thousands of eclipsing binaries, the European Space Agency PLATO mission will (if selected to fly) provide a much greater banquet. Compared to its immediate predecessors, PLATO is envisaged to have a wider sky coverage $\left(500 \mathrm{deg}^{2}\right)$, a larger number of target stars $(400000)$, and a much improved sampling rate $(25 \mathrm{~s})$. It will also aim for brighter stars on average, allowing follow-up observations to be obtained from many ground-based telescopes. PLATO will have a working group dedicated to studying eclipsing binaries: contact me if you are interested!

\section{References}

Borucki, W. J., et al., 2010, Science, 327, 977

Bruntt, H. \& Southworth, J., 2007, IAU Symp. 240, 624

Bruntt, H. \& Southworth, J., 2008, JPhys. Conf. Ser., 118, 012012

Bruntt, H., et al., 2006, A\&SA, 456, 651

Carter, J. A., et al., 2011, Science, 331, 562

Cherepashchuk, A., Eaton, J. A., \& Khaliullin, Kh. F. 1984, ApJ, 281, 774

Derekas, A., et al., 2011, Science, 332, 216

Hekker, S., et al., 2010, ApJ, 713, L187

Kondo, Y., McCluskey, G. E., Silvis, J. M. S., Polidan, R. S., McCluskey, C. P. S., \& Eaton, J. A. $1994, A p J, 421,787$

Maceroni, C., et al., 2009, A\&A, 508, 1375

Perryman, M. A. C., et al., 1997, A\&A, 323, L49

Pribulla, T., et al., 2010, AN, 331, 397

Southworth, J. Bruntt, H., \& Buzasi, D. L. 2007, A\&A, 467, 1215

Southworth, J., et al., 2011, MNRAS, 414, 2413

Southworth, J., et al., 2011, MNRAS, 414, 3740

Stebbins, J., 1911, ApJ, 34, 112 